\title{
3 Research Suare

\section{Evaluation of The Effect of Epiphytic, Endophytic And Rhizosphere Bacteria On Seed Germination And Seedling Characteristics}

Mohammad Razinataj ( $\sim$ m.razi@areeo.ac.ir)

Cotton Research Institute

Gholam Khodakaramian

Bu Ali Sina University Faculty of Agriculture

\section{Research}

Keywords: endophytic, rhizosphere, cotton, bacteria

Posted Date: June 9th, 2021

DOI: https://doi.org/10.21203/rs.3.rs-541689/v1

License: (c) (i) This work is licensed under a Creative Commons Attribution 4.0 International License.

Read Full License 


\section{Abstract}

During 2013 and 2014, in order to investigate the effect of bacteria on the characteristics and growth rate of cotton seedlings, isolated epiphytic, endophytic and rhizospheric bacteria from cotton plants in Golestan province. Based on biochemical and phenotypic tests, amplification and sequencing of $16 S$ rRNA gene, were identified strains. Isolates of Bacillus pumilus MR11, B. pumilus MR12, B. pumilus MR13, B. safensis MR21, B. safensis MR22 and Stenotrophomonas pavanii MR31 identified as superior endophytes and rhizosphere. Also Pseudomonas fluorescens, $P$. syringae and Pantoea annanatis were identified as epiphytic from plants and seeds. These isolates were evaluated to effect on seedling growth characteristics. Based on results, the six-day-old seedlings treated with Bacillus pumilus MR11 and $B$. pumilus MR12 had the highest root and shoot length and fresh shoot weight. Due to the fact that $B$. pumilus MR12 and $S$. pavanii MR31 isolates had the highest percentage and germination rate, so their use as seed treatment can increase the percentage and germination rate of seeds and be effective in reducing the damage caused by seedling diseases. This is the first report of isolation of $B$. safensis, $B$. pumilus and S. pavanii bacteria from rhizosphere and cotton endophytes in Iran.

\section{Introduction}

Phylosphere is the surface of the aerial parts of plants and a place for micro-organisms to live. Simple sugars such as glucose, fructose and sucrose are the predominant carbonaceous substances on leaves and stem that pass out of wound areas or secretory pores. In these areas, can be seen the largest population bacteria of phylosphere (Mercier and Lindow 2000). The microbial inhabitants of the phylosphere include different genera of bacteria, filamentous fungi, yeasts, algae, protozoa and nematodes. The bacteria are the most abundant inhabitants of the phylosphere (Andrews and Harris 2000). Plant species and leaf type have a beneficial effect on the number of bacteria of phylosphere. For example, the number of bacteria in the phylosphere of broadleaf plants such as cucumbers and beans is significantly higher than that of broadleaf plants with waxy leaves and grasses. On the other hand, the surface of the aerial parts of the plant is subject to rapid and continuous changes in temperature, humidity, ultraviolet rays, relative humidity and the concentration gradient of nutrients (Brencic and Winans 2005).

The rhizosphere is an area of the root that soil microorganisms are very interested in, using root secretions as carbon and energy sources and competing for food and trritory. Bacteria such as Bacillus, Pseudomonas, Azospirillium, Azotobacter, Arthrobacter, Entrobacter and Serratia are among the growthpromoting bacteria (Podile and Kishore 2006). Plant growth-promoting bacteria are part of the integrated disease management program and reduce chemical consumption (Shivalingaiah et al. 2013). These bacteria can stimulate the induction resistance system against a wide range of plant pathogens (Pieterse et al. 2003). The induction of diseases resistance in various crops such as banana, beans, rice, and cucumbers has been reported by Pseudomonas and Bacillus (Harish et al. 2008; Hasan et al. 2010). Most Pseudomonas spp. significantly increases plant length, root length, and dry matter, leading to the production of plant buds and roots. Most plant growth-promoting bacteria are able to settle in the roots, 
especially if inoculated before sowing. The use of plant growth promoting bacteria as a biological control agent is an alternative to the use of chemical fertilizers that have led to environmental pollution (Ali et al. 2010). Pseudomonads are the major group of the plant growth-promoting bacteria, which act by strategies such as the production of plant hormones, stimulating nutrient uptake, and controlling plant pathogens (Minaxi 2010).

Bacillus species were reported as endophytes within different plant tissues. $B$. megaterium was the most abundant species in the rhizosphere and $B$. pumilus and $B$. subtilis were the most abundant species in the soybean phylosphere (Arias et al. 1999). B. endophyticus from the inner tissue of cotton fibers (Reva et al, 2002), Erwinia sp., Bacillus sp., B. pumilus, B. brevis, Clavibacter sp. and Xanthomonas sp. isolated as endophytes from roots, stems, buds and bolls (Misaghi and Donndelinger 1990). Enterobacter asburiae was isolated as an endophyte and from the cotton rhizosphere, which showed that this bacterium is able to spread systemically in the plant (Hallmann et al. 1998). P. fluorescens caused a significant increase in germination and dry weight of cotton in greenhouse conditions (Salaheddin et al. 2010). P. fluorescens stimulated seedling growth and increased yield with reduced disease (Safyazov et al. 1995).

\section{Materials And Methods}

During the growth period of cotton plant in the years 2013-2014, random sampling was done from different aerial parts and plant roots in different regions of Golestan province in Iran. In order to study bacteria of rhizosphere, the plants were transferred to the laboratory along with the roots and soil in paper bags.

\section{Isolation of bacteria and study of the characteristics of the strains}

The bacteria isolated from rhizosphere (Ahmad et al. 2008), endophyte (Misaghi and Donndelinger 1990) and epiphytes (Mehta et al., 2005). After purification, the phenotypic and biochemical characteristics of isolates were determined based on valid bacteriological references (Fahy, and Persley 1983; Schaad et al. 2001).

\section{Evaluation of the effect of bacteria on seedling growth}

The seeds were soaked in $10^{6} \mathrm{CFU}$ dilution bacterial suspension for 10 minutes, then 100 seeds were sown in 3 repetitions in Whatman paper and incubated at $25^{\circ} \mathrm{C}$ with a period of 16 hours of light and 8 hours of darkness. Number of germinated seeds counted on the third and sixth days and 10 seeds randomly selected in each treatment and were measured characteristics such as root and shoot growth length, fresh and dry root weight, stem fresh and dry weight, total fresh and dry weight, amount of root, stem and total tissue water. To obtain the dry weight of stem and root, the samples were placed in an oven at $75^{\circ} \mathrm{C}$ for 72 hours and then were recorded data (ISTA 2015). 


\section{Identification of selected bacteria using molecular studies}

The taxonomic position of the selected bacteria was assessed by determining the sequence of the $16 \mathrm{~s}$ rRNA gene region. To propagate this region, CinnaGen Company primers were used with sequences F27: 5'-AGAGTTTGATCMTGGCTCAG-3' and R1492: 5'-TACGGYTACCTTGTTACGACTT-3' (Lane 1991). For DNA extraction, bacterial isolates were cultured on Nutrient Agar medium for 24 hours after growth at $28^{\circ}$ C. Bacterial cells were suspended in sterile distilled water and the turbidity of the suspension was adjusted to $0.1-1.2$ units at $600 \mathrm{~nm}$. To each sample was added 0.1 volume of normal potassium hydroxide. The samples were boiled for 10 minutes and then centrifuged at 13,000 rpm for 5 minutes. The top of solution was separated and used as a DNA-containing solution for some genotypic tests (Arabi et al, 2006). To amplify this gene region using PCR, the reaction was performed at a final volume of $25 \mu$ as follows:

The reaction mixture consisted of $2 \mu \mathrm{l}$ of DNA, $2.5 \mu$ of PCR buffer $1 \mathrm{X}, 1 \mu \mathrm{mol}$ of each primer, $0.2 \mathrm{mmol}$ of dNTPs, $1.5 \mu \mathrm{mol}$ of magnesium chloride and 3 units of Taq polymerase and a water content of 0.9517 $\mu \mathrm{l}$. Temporal and temperature conditions included the initial step of denaturation at $94^{\circ} \mathrm{C}$ for 5 minutes and then 35 separate cycles (including denaturation at $94^{\circ} \mathrm{C}$ for one minute, anealing at one minute in $55^{\circ} \mathrm{C}$ and DNA extension at $72^{\circ} \mathrm{C}$ for 90 seconds) and finally, a final elongation step at $72^{\circ} \mathrm{C}$ for 10 minutes (Ausubel et al. 1992). After confirmation of the presence of amplification band in $1.5 \%$ agarose gel in PCR product, amplified samples were sent to Macrogen (South Korea) for purification and sequencing.

Bioedit 7.0.9.0 software was used for multiple alignments of the sequences obtained from the studied isolates. Mega 5 software was used to compare the similarity and genetic distance of sequences (Felsenstein 1989). Phylogenetic tree was calculated by Neighbor-Joining method and Jukes-Cantor distance matrix with 1000 Bootstrap replications and $E$. coli were used for gram-negative bacteria and Paenibacillus bracinonensis for gram positive bacteria as an out group in drawing (Jukes and Cantor 1969) and Tree View 1.6.6 (Page 1996) was used to observe and analyze it.

\section{Results And Discussion}

Based on the comparison of characteristics means, the six-day-old seedlings of B. pumilus MR11 and $B$. pumilus MR12 had the highest root and shoot length and shoot fresh weight. They were also superior to other characrteristics (Tables 2 and 3). B. pumilus MR12 and S. pavanii MR31 isolates had the highest percentage and germination rate, so its use as seed treatment can increase the percentage and germination rate of seeds and reduce the amount of seedling diseases. Based on the results of $16 S$ rRNA gene amplification and phenotypic characteristics, were identified isolates of $S$. pavanii MR31, B. safensis MR21, B. safensis MR22, B. pumilus MR13, B. pumilus MR12 and B. pumilus MR11 (Fig. 1 and Table 1). 
Table 1

Phenotypic and biochemical characteristics of bacterial isolates from rhizosphere and endophyte of cotton.

\begin{tabular}{|c|c|c|c|c|c|c|}
\hline $\begin{array}{l}\text { Isolate } \\
\text { Test }\end{array}$ & $\begin{array}{l}\text { S. pavanii } \\
\text { MR31 }\end{array}$ & $\begin{array}{l}\text { B. pumilus } \\
\text { MR13 }\end{array}$ & $\begin{array}{l}\text { B. pumilus } \\
\text { MR12 }\end{array}$ & $\begin{array}{l}\text { B. pumilus } \\
\text { MR11 }\end{array}$ & $\begin{array}{l}\text { B. } \\
\text { safensis } \\
\text { MR22 }\end{array}$ & $\begin{array}{l}\text { B. safensis } \\
\text { MR21 }\end{array}$ \\
\hline Gram reaction & - & + & + & + & + & + \\
\hline Oxidase & - & - & - & - & - & - \\
\hline $\begin{array}{l}\text { Flourescent on } \\
\text { KB medium }\end{array}$ & - & - & - & - & - & - \\
\hline $\begin{array}{l}\text { Oxidative } \\
\text { metabolism }\end{array}$ & + & + & + & + & + & + \\
\hline $\begin{array}{l}\text { Fermentative } \\
\text { metabolism }\end{array}$ & - & - & - & - & - & - \\
\hline $\begin{array}{l}\text { Soft rot on } \\
\text { potato }\end{array}$ & - & - & - & - & - & - \\
\hline $\begin{array}{l}\text { Starch } \\
\text { hydrolysis }\end{array}$ & - & - & - & - & - & - \\
\hline Nitrate reduction & - & - & - & - & - & - \\
\hline $\begin{array}{l}\text { Gelatin } \\
\text { hydrolysis }\end{array}$ & - & + & + & + & + & + \\
\hline Lipase & - & - & - & - & - & - \\
\hline $\begin{array}{l}\text { Growth on } \mathrm{NaCl} \\
3 \%\end{array}$ & + & + & + & + & + & + \\
\hline $\begin{array}{l}\text { Growth on } \mathrm{NaCl} \\
5 \%\end{array}$ & - & + & + & + & + & + \\
\hline \multicolumn{7}{|l|}{ Utilization of: } \\
\hline Glucose & + & + & + & + & + & + \\
\hline Sucrose & + & + & + & - & + & + \\
\hline D-mannose & + & + & + & + & + & + \\
\hline D-galactose & + & + & + & + & + & + \\
\hline Cellobiose & + & + & + & - & - & + \\
\hline Arabinose & + & - & + & - & - & + \\
\hline D-fructose & + & - & + & + & + & + \\
\hline Trehalose & + & + & + & + & + & + \\
\hline
\end{tabular}




\begin{tabular}{|lllllll|}
\hline Isolate & $\begin{array}{l}\text { S.pavanii } \\
\text { Mest }\end{array}$ & $\begin{array}{l}\text { B.pumilus } \\
\text { MR13 }\end{array}$ & $\begin{array}{l}\text { B. pumilus } \\
\text { MR12 }\end{array}$ & $\begin{array}{l}\text { B. pumilus } \\
\text { MR11 }\end{array}$ & $\begin{array}{l}\text { B. } \\
\text { safensis } \\
\text { MR22 }\end{array}$ & $\begin{array}{l}\text { B. safensis } \\
\text { MR21 }\end{array}$ \\
\hline Acetate & - & - & - & - & - & - \\
\hline Malonate & - & + & + & - & - & - \\
\hline L-tartrate & - & - & + & - & + & + \\
\hline Urate & - & - & - & - & - & - \\
\hline Citrate & + & - & - & - & - & - \\
\hline D-galactorunate & - & + & - & - & - & - \\
\hline L-maleate & - & - & - & - & - & - \\
\hline Lactate & + & + & - & + & + & - \\
\hline Nicutinate & - & - & - & - & - & - \\
\hline Meso-inositol & - & + & - & + & - & - \\
\hline L-cysteine & - & - & - & - & - & + \\
\hline Ganine & - & - & - & - & - & + \\
\hline Glysine & + & + & + & + & + & + \\
\hline Caseine & + & + & + & + & + & + \\
\hline
\end{tabular}


Table 2

The Comparison of different characteristics means of three-day cotton seedling in seed treatments representing cotton growth bacteria.

\begin{tabular}{|c|c|c|c|c|c|}
\hline $\begin{array}{l}\text { characteristics } \\
\text { Isolate }\end{array}$ & $\begin{array}{l}\text { Seedling wet } \\
\text { weight (mg) }\end{array}$ & $\begin{array}{l}\text { Seedling dry } \\
\text { weight (mg) }\end{array}$ & $\begin{array}{l}\text { Pedicle } \\
\text { length (cm) }\end{array}$ & $\begin{array}{l}\text { Radicle } \\
\text { length (cm) }\end{array}$ & $\begin{array}{l}\text { Pedicle wet } \\
\text { weight (mg) }\end{array}$ \\
\hline Control & $510 \mathrm{cde}$ & $300 \mathrm{a}$ & $1.31 \mathrm{a}$ & $2.3 \mathrm{c}$ & $445 a$ \\
\hline $\begin{array}{l}\text { B..pumilus MR } \\
11\end{array}$ & $485 \mathrm{de}$ & $282 a b$ & $1.23 \mathrm{a}$ & $2.01 \mathrm{~cd}$ & $427 \mathrm{a}$ \\
\hline $\begin{array}{l}\text { B. pumilus } \\
\text { MR13 }\end{array}$ & $590 \mathrm{bcd}$ & $285 a b$ & $1.37 \mathrm{a}$ & $2.73 \mathrm{bc}$ & $470 a$ \\
\hline P. fluorescens & $482 \mathrm{e}$ & $250 \mathrm{~b}$ & $1.35 \mathrm{a}$ & $1.44 \mathrm{~d}$ & $419 a$ \\
\hline $\begin{array}{l}\text { B. pumilus } \\
\text { MR12 }\end{array}$ & 550 cde & $270 a b$ & $1.15 \mathrm{a}$ & $2.48 \mathrm{c}$ & $420 \mathrm{a}$ \\
\hline B. pumilus & $657 a b$ & $287 a b$ & $1.33 \mathrm{a}$ & $3.25 a b$ & $490 \mathrm{a}$ \\
\hline P. annanatis & $535 \mathrm{cde}$ & $285 a b$ & $1.2 \mathrm{a}$ & $2.1 \mathrm{~cd}$ & $460 \mathrm{a}$ \\
\hline P. annanatis & 570 b-e & $280 \mathrm{ab}$ & $1.25 \mathrm{a}$ & $2.5 \mathrm{c}$ & $460 a$ \\
\hline $\begin{array}{l}\text { B. safensis } \\
\text { MR22 }\end{array}$ & 605 bc & $265 a b$ & $1.3 \mathrm{a}$ & $3.51 \mathrm{a}$ & $457 a$ \\
\hline $\begin{array}{l}\text { B. safensis } \\
\text { MR21 }\end{array}$ & 532 cde & $265 a b$ & $1.3 \mathrm{a}$ & $2.31 \mathrm{c}$ & $430 \mathrm{a}$ \\
\hline P. syringae & 532 cde & $282 a b$ & $1.3 \mathrm{a}$ & $2.23 \mathrm{c}$ & $452 \mathrm{a}$ \\
\hline $\begin{array}{l}\text { S. pavanii } \\
\text { MR31 }\end{array}$ & 537 cde & $275 a b$ & $1.29 a$ & $2.21 \mathrm{c}$ & $450 \mathrm{a}$ \\
\hline P. fluorescens & 595 bc & $280 a b$ & $1.28 \mathrm{a}$ & $2.14 \mathrm{~cd}$ & $485 a$ \\
\hline P. syringae & $705 a$ & $267 \mathrm{ab}$ & $1.31 \mathrm{a}$ & $3.26 \mathrm{ab}$ & $487 a$ \\
\hline
\end{tabular}


Table 2

Continued.

\begin{tabular}{|c|c|c|c|c|}
\hline $\begin{array}{l}\text { characteristics } \\
\text { Isolate }\end{array}$ & $\begin{array}{l}\text { Radicle wet } \\
\text { weight (mg) }\end{array}$ & Pedicle dry weight (mg) & $\begin{array}{l}\text { Radicle dry } \\
\text { weight } \\
\text { (mg) }\end{array}$ & $\begin{array}{l}\text { Germination } \\
\text { percentage }\end{array}$ \\
\hline Control & 65 ef & 290 a & $10 \mathrm{c}$ & $93 \mathrm{bcd}$ \\
\hline $\begin{array}{l}\text { B..pumilus MR } \\
11\end{array}$ & $57 \mathrm{f}$ & $272 \mathrm{a}$ & $10 \mathrm{c}$ & 84 ef \\
\hline $\begin{array}{l}\text { B. pumilus } \\
\text { MR13 }\end{array}$ & 122 b-e & $275 a$ & $10 \mathrm{c}$ & $92 \mathrm{~cd}$ \\
\hline P. fluorescens & $62 f$ & $242 \mathrm{a}$ & $7 c$ & $94 \mathrm{bc}$ \\
\hline $\begin{array}{l}\text { B. pumilus } \\
\text { MR12 }\end{array}$ & $127 \mathrm{bcd}$ & $252 \mathrm{a}$ & $17 a b$ & 97 a \\
\hline B. pumilus & $167 b$ & $272 \mathrm{a}$ & $17 a b$ & $95 a b$ \\
\hline P. annanatis & 82 def & 277 a & $10 \mathrm{c}$ & 86 e \\
\hline P. annanatis & $107 c-f$ & $107 c-f$ & $12 \mathrm{bc}$ & $95 \mathrm{ab}$ \\
\hline $\begin{array}{l}\text { B. safensis } \\
\text { MR22 }\end{array}$ & $147 \mathrm{bc}$ & $147 \mathrm{bc}$ & $20 \mathrm{a}$ & $91 \mathrm{~d}$ \\
\hline $\begin{array}{l}\text { B. safensis } \\
\text { MR21 }\end{array}$ & $102 c-f$ & $102 c-f$ & $10 \mathrm{c}$ & $95 a b$ \\
\hline P. syringae & 77 def & 77 def & $10 \mathrm{c}$ & $82 \mathrm{f}$ \\
\hline $\begin{array}{l}\text { S. pavanii } \\
\text { MR31 }\end{array}$ & $90 c-f$ & $90 c-f$ & $10 \mathrm{c}$ & $95 a b$ \\
\hline P. fluorescens & $112 b-f$ & $112 b-f$ & $10 \mathrm{c}$ & 86 e \\
\hline P. syringae & $220 \mathrm{a}$ & $220 a$ & $17 a b$ & $92 \mathrm{~cd}$ \\
\hline
\end{tabular}


Table 3

The Comparison of different characteristics means of six-day cotton seedling in seed treatments representing cotton growth bacteria.

\begin{tabular}{|c|c|c|c|c|c|}
\hline $\begin{array}{l}\text { characteristics } \\
\text { Isolate }\end{array}$ & $\begin{array}{l}\text { Seedling wet } \\
\text { weight (mg) }\end{array}$ & $\begin{array}{l}\text { Seedling dry } \\
\text { weight (mg) }\end{array}$ & $\begin{array}{l}\text { Radicle } \\
\text { length (cm) }\end{array}$ & $\begin{array}{l}\text { Pedicle } \\
\text { length }(\mathrm{cm})\end{array}$ & $\begin{array}{l}\text { Pedicle wet } \\
\text { weight (mg) }\end{array}$ \\
\hline Control & $948 \mathrm{~cd}$ & $287 \mathrm{abc}$ & $2.74 \mathrm{f}$ & $4.67 \mathrm{de}$ & $937 \mathrm{bc}$ \\
\hline $\begin{array}{l}\text { B.pumilus } \\
\text { MR11 }\end{array}$ & 1180 a-d & $270 a b c$ & $6.67 a$ & $6.76 a$ & $1150 \mathrm{ab}$ \\
\hline $\begin{array}{l}\text { B. pumilus } \\
\text { MR13 }\end{array}$ & $1050 \mathrm{bcd}$ & $240 \mathrm{c}$ & $5.95 \mathrm{ab}$ & 5.33 a-e & $867 c$ \\
\hline P. fluorescens & 1140 a-d & $290 \mathrm{abc}$ & $2.67 \mathrm{f}$ & 4.98 cde & $1127 a b$ \\
\hline $\begin{array}{l}\text { B. pumilus } \\
\text { MR12 }\end{array}$ & 1160 a-d & $242 c$ & $5.7 \mathrm{abc}$ & $6.25 \mathrm{abc}$ & $1117 a b$ \\
\hline B. pumilus & $944 d$ & $255 \mathrm{bc}$ & $5.2 \mathrm{bc}$ & $5.15 b-e$ & $920 \mathrm{bc}$ \\
\hline P. annanatis & $1040 \mathrm{bcd}$ & $270 \mathrm{abc}$ & 3.73 def & $5.21 \mathrm{~b}-\mathrm{e}$ & 1000 bc \\
\hline P. annanatis & $1060 \mathrm{bcd}$ & $257 \mathrm{bc}$ & $4.95 \mathrm{bcd}$ & 5.55 a-e & $104 \mathrm{bc}$ \\
\hline $\begin{array}{l}\text { B. safensis } \\
\text { MR22 }\end{array}$ & 1090 a-d & $255 \mathrm{bc}$ & 4.4 cde & $4.76 \mathrm{de}$ & 952 bc \\
\hline $\begin{array}{l}\text { B. safensis } \\
\text { MR21 }\end{array}$ & 1170 a-d & $330 \mathrm{a}$ & $4.85 \mathrm{bcd}$ & $5.3 \mathrm{~b}-\mathrm{e}$ & $957 \mathrm{bc}$ \\
\hline P. syringae & $1330 \mathrm{a}$ & $275 a b c$ & 3.37 ef & $6.59 \mathrm{ab}$ & $1370 \mathrm{a}$ \\
\hline $\begin{array}{l}\text { S. pavanii } \\
\text { MR31 }\end{array}$ & 1190 abc & $317 a b$ & $5.46 \mathrm{abc}$ & $4.1 \mathrm{e}$ & $980 \mathrm{bc}$ \\
\hline P. fluorescens & $963 \mathrm{bcd}$ & $295 a b c$ & $4.56 b-f$ & $4.75 \mathrm{de}$ & 940 bc \\
\hline P. syringae & $1200 a b$ & $262 a b c$ & $5 \mathrm{bcd}$ & 5.79 a-d & $1050 \mathrm{abc}$ \\
\hline
\end{tabular}


Table 3

Continued.

\begin{tabular}{|c|c|c|c|c|}
\hline $\begin{array}{l}\text { characteristics } \\
\text { Isolate }\end{array}$ & $\begin{array}{l}\text { Radicle wet } \\
\text { weight (mg) }\end{array}$ & $\begin{array}{l}\text { Pedicle dry } \\
\text { weight (mg) }\end{array}$ & $\begin{array}{l}\text { Radicle dry weight } \\
\text { (mg) }\end{array}$ & $\begin{array}{l}\text { Germination } \\
\text { percentage }\end{array}$ \\
\hline Control & $14 \mathrm{ef}$ & $282 \mathrm{abc}$ & $4 \mathrm{fg}$ & $96 b$ \\
\hline $\begin{array}{l}\text { B..pumilus } \\
\text { MR11 }\end{array}$ & $34 b-f$ & $256 a b c$ & $12 a b$ & $96 b$ \\
\hline $\begin{array}{l}\text { B. pumilus } \\
\text { MR13 }\end{array}$ & $33 c-f$ & $228 \mathrm{c}$ & $11 \mathrm{abc}$ & $93 \mathrm{c}$ \\
\hline P. fluorescens & 19 ef & $285 a b c$ & $3 \mathrm{~g}$ & $98 a b$ \\
\hline $\begin{array}{l}\text { B. pumilus } \\
\text { MR12 }\end{array}$ & $42 \mathrm{bcd}$ & $233 c$ & 9 a-d & $98 a b$ \\
\hline B. pumilus & $25 c-f$ & $245 \mathrm{bc}$ & 9 a-d & $96 \mathrm{~b}$ \\
\hline P. annanatis & $45 a b c$ & $265 a b c$ & 5 efg & $87 d$ \\
\hline P. annanatis & 19 ef & $251 \mathrm{bc}$ & 7 def & $100 \mathrm{a}$ \\
\hline $\begin{array}{l}\text { B. safensis } \\
\text { MR22 }\end{array}$ & $47 a b$ & $243 \mathrm{bc}$ & 10 a-d & $98 a b$ \\
\hline $\begin{array}{l}\text { B. safensis } \\
\text { MR21 }\end{array}$ & $11 \mathrm{f}$ & 323 a & $8 \mathrm{cde}$ & $96 \mathrm{~b}$ \\
\hline P. syringae & $59 a$ & $270 a b c$ & $5 \mathrm{efg}$ & $96 \mathrm{~b}$ \\
\hline $\begin{array}{l}\text { S. pavanii } \\
\text { MR31 }\end{array}$ & $58 \mathrm{a}$ & $305 a b$ & $13 \mathrm{a}$ & $100 \mathrm{a}$ \\
\hline P. fluorescens & 21 def & $287 \mathrm{abc}$ & 7 def & $92 \mathrm{c}$ \\
\hline P. syringae & 35 b-e & $252 b c$ & 7 def & $100 \mathrm{a}$ \\
\hline
\end{tabular}


Table 4

Characteristics of the bacteria isolates based on 16S rRNA gene sequence.

\begin{tabular}{|llll|}
\hline Isolate & Accession numbers at NCBI & Closest type strain & Similarity \\
\hline B. pumilus MR11 & $\mathrm{KY} 067432.1$ & B. pumilus AB020208.1 & 99 \\
\hline B. pumilus MR12 & $\mathrm{KY} 067433.1$ & B. pumilus AB020208.1 & 98 \\
\hline B. pumilus MR13 & $\mathrm{KY} 067435.1$ & B. pumilus AB020208.1 & 99 \\
\hline B. safensis MR21 & $\mathrm{KY} 067431.1$ & B. safensis NR-113945.1 & 99 \\
\hline B. safensis MR22 & $\mathrm{KY} 067434.1$ & B. safensis NR-113945.1 & 99 \\
\hline S. pavanii MR31 & $\mathrm{KY} 067436.1$ & S. pavanii FJ748683.2 & 99 \\
\hline
\end{tabular}

MR31 isolate was not able to reduce nitrate, contrary to the results of Brenner et al. (2006) (Table 1). Based on the biochemical properties of MR31 isolate was identified as Stenetrophomonas sp. and based on the results of $16 S$ rRNA gene analysis identified S. pavanii. The isolates MR11, MR12 and MR13 were consistent with Brenner et al. (2006) by using arabinose, fructose, cellobiose, galactose and mesoinositol. $B$. safensis can be differed from $B$. pumilus by acid production from inositol, maltose, D-turanose, methyl alpha-d-glucopyranoside and the utilization of inositol, and negative in lipase and casein hydrolysis of $B$. pumilus (Sotami et al. 2006). Based on phenotypic characteristics, biochemical and molecular studies of 16S rRNA gene (Table 4), MR11, MR12 and MR13 isolates were identified as B. pumilus and MR21 and MR22 isolates were identified as $B$. safensis. B. pumilus was isolated endophytically from cotton roots, stems, buds and bolls (Misaghi and Donndelinger 1990). Based on phenotypic characteristics, biochemical tests and sequencing of $16 S$ rRNA gene and gyrase $B$ gene, were identified $B$. pumilus (Kavaleva et al. 2015) and B. safensis (Fonseca et al. 2015). Based on the phylogenetic relationship, $B$. pumilus and $B$. safensis were in one group that was consistent with Kakade and Chaphalkar (2017). Similarities of 1500 bp sequence of $16 S$ rRNA gene in S. pavanii MR31 isolate with FJ748683.1 gene bank isolate, $B$. safensis MR21 and $B$. safensis MR22 isolates with NR113945.1 gene bank and $B$. pumilus MR13 and B. pumilus MR11 were $99 \%$ similar to AB020208.1 gene bank isolates and B. pumilus MR12 isolates was 98\% similar to isolates of AB020208.1 gene bank (Figs. 2 and 3). Based on the effect of eight isolates of Bacillus sp. against seven isolates of fungi causing cotton seedling disease, was observed a very significant relationship in characteristics such as pre- and post-vegetative seedling diseases, survival, height and dry weight of the plant (Khiyami et al. 2014). The use of $P$. fluorescens had significant effects on increasing germination, vigor and dry weight of cotton seedlings under greenhouse conditions. This isolate stimulated seedling emergence and growth and increased yield with reduction of disease (Safyazov et al. 1995). Root and sprout length of tomatoes, cucumbers, lettuce and potatoes increased by using of Pseudomonas sp. (VanPeer and Schippers 1988). There is less knowledge in Bacillus sp. compared with Pseudomonas sp. while they are as typical soil bacteria, play an important role in plant growth and bio-control (Kloepper et al. 2004b). 


\section{Declarations}

Authors' contributions: Razinataj, M. wrote the initial draft of the manuscript. Khodakaramian, G. made all necessary corrections and carried out final editing of manuscript.

Funding: This work was supported by Cotton Research Institute of Iran and Bu-Ali-Sina University.

Availability of data and materials are applicable.

Ethics approval and consent: Dear Editor in chief, we wish to submit a new manuscript entitled "Evaluation of the effect of epiphytic, endophytic and rhizosphere bacteria on seed germination and seedling characteristics" for consideration by the Journal of Cotton Research. We do not confirm that this work is original and has not been published elsewhere nor is it currently under consideration for publication elsewhere.

Consent for publication not applicable.

Competing interests Authors declare that they have no conflict of interest for the publication of the manuscript.

Author details:

1- Cotton Reserch Institute of Iran, Agricultural Research, Education and Extension Organization (AREEO), Gorgan, Iran.

2- 2- Faculty of Agriculture, Bu-Ali Sina University, Hamedan, Iran.

Acknowledgements: My special thanks are extended to staff of Cotton Research Institute of Iran.

\section{References}

1. Ahmad F, Ahmad I, Khan MS. 2008. Screening of free-living rhizospheric bacteria for their multiple plant growth promoting activities. Microbiological Research. 163:173-181.

2. Ali B, Sabri AN, Hasnain S. 2010. Rhizobacterial potential to alter auxin content and growth of Vigna radiata (L.). World Journal of Microbiology and Biotechnology. 26:1379-1384.

3. Andrews LH, Harris RF. 2000. The ecology and biogeography of microorganisms on plant surfaces. Annual Review of Phytopathology. 38:145-180.

4. Arias RS, Sagardoy MA, vanVuurde JWL. 1999 Spatio-temporal distribution of naturally occurring Bacillus spp. and other bacteria on the phylloplane of soybean under field conditions. Journal of Basic Microbiology. 39:283-292.

5. Ausubel FM, Brent R, Kingston RE, Moore DD, Seidman JG, Smith JA, Struhl K. 1992. "Current Protocols in Molecular Biology." (New York: Greene Publishing Association; Wiley-Interscience). 
6. Brencic A, Winans SC. 2005. Detection of and response to signals involved in host-microbe interaction by plant-associated bacteria. Microbiology and Molecular Biology Reviews. 69:155-194.

7. Brenner DJ, Krieg NR, Staley JT, Garrity GM. 2006. "Bergy's Mannual of Systematic Bacteriology, Gammaproteobacteria. $2^{\text {nd }}$ ed. Vol 2, Part B." 1106 pp. (Springer)

8. Fahy PC, Persley GJ. 1983. "Plant Bacterial Disease: A Diagnostic Guide.” (Academic Press Sydney $393 \mathrm{pp})$.

9. Felsenstein J. 1989. PHYLIP-Phylogeny inference package (Ver. 3.2). Cladistics. 5:164-166.

10. Fonseca FSA, Angolini CFF, Arruda MAZ, Junior CAL, Santos CA, Saraiva AM, Pilau E, Souza AP, Laborda PR, deOliveira PFL, deOliveira VM, Reis FAM, Marsaioli AJ. 2015. Identification of oxidoreductases from the petroleum Bacillus safensis strain. Biotechnology Reports. 8:152-159.

11. Hallmann J, Quadt-Hallmann A, Rodriguez-Kabana R, Kloepper JW. 1998. Interactions between Meloidogyne incognita and endophytic bacteriain cotton and cucumber. Soil Biology and Biochemistry. 30:925-937.

12. Harish S, Kavino M, Kumar N, Saravanakumar D, Soorianathasundaram K, Samiyappan R. 2008. Biohardening with plant growth promoting rhizosphere and endophytic bacteria induces systemic resistance against Banana bunchy top virus. Applied Soil Ecology. 39:187-200.

13. Hasan MN, Afghan S, Hafeez FY. 2010. Suppression of red rot caused by Coletotrichium falcatum on sugarcane plants using plant growth-rhizobacteria. Bio Control. 55:531-542.

14. ISTA. 2015. "Handbook for International Rules for Seed Testing." (International Seed Testing Association, Zurich, Switzerland, 276 pp.)

15. Jukes TH, Cantor CR. 1969. Evolution of protein molecules. In "Mammalian Protein Metabolism". (Ed HN, Munro) pp. 21-132. (Academic Press, New York).

16. Kakade PD, Chaphalkar SR. 2017. Isolation and purification of antibacterial peptide from Bacillus safensis, endophytica bacteria from Anthocephalus kadamba. International Journal of Current Microbiology and Applied Sciences. 6:504-511.

17. Khiyami MA, Omar MR, Abd-Elsalam KA, Aly AA. 2014. Bacillus-based biological control of cotton seedling disease complex. Journal of Plant Protection Research. 54:340-348.

18. Kloepper JW, Reddy MS, Kenney DS, Vavrina C, Kokalis-Burelle N, Martinez-Ochoa N. 2004b. Theory and applications of rhizobacteria for transplant production and yield enhancement. (Nicola S, Nowak $\mathrm{J}$, Vavrina CS, editors. Proceedings of the XXVI IHC - transplant production and stand establishment. Acta Horticulture. 631:217-229.

19. Lane DJ. 1991. 16S/23S rRNA sequencing. In "Nucleic acid techniques in bacterial systematics". (Eds E Stackebrandt, M Goodfellow) pp 115-175. (John Wiley \& Sons, Ltd. Chichester, England).

20. Mehta YR, Boonfeti C Bolognini V. 2005. A semi-selective agar medium to detect the presence of Xanthomonas axonopodis pv. malvacearum naturally infected cotton seed. Fitopatologia Brasileira. 30:489-496. 
21. Mercier J, Lindow SE. 2000. Role of leaf surface sugars in colonization of plants by bacterial epiphytes. Applied and Environmental Microbiology. 66:369-374.

22. Minaxi SJ. 2010. Disease suppression and crop improvement in moong beans (Vigna radiata) through Pseudomonas and Burkholderia strains isolated from semi-arid region of Rajasthan, India. Bio Control. 55:799-810.

23. Misaghi IJ, Donndelinger CR. 1990. Endophytic bacteria in symptom-free cotton plants. Phytopathology. 80:808-811.

24. Page RDM. 1996. TREEVIEW: An application to display phylogenetic trees on personal computers. Computer Applications in the Biosciences. 12:357-358.

25. Pieterse CMJ, Pelt JA, Verhagen BWM, Jurriaan T, Wees SCM. Léon-Kloosterziel KM, Loon, LC. 2003. Induced systemic resistance by plant growth-promoting rhizobacteria. Symbiosis. 35:39-54.

26. Podile AR, Kishore GK. 2006. Plant growth promoting rhizobacteria. In "Plant Associated Bacteria" (Ed SS Gnanamanickam). pp. 195-230. (Springer Publishers, The Netherlands,).

27. Reva ON, Smirnov VV, Pettersson B, Priest FG. 2002. Bacillus endophyticus sp. nov., isolated from the inner tissues of cotton plants (Gossypium sp.). International Journal of Systematic and Evolutionary Microbiology. 52:101-107.

28. Safyazov JS, Mannanov RN, Sattarova RF. 1995. The use of bacterial antagonists for the control of cotton diseases. Field Crops Research. 43:51-54.

29. Salaheddin K, Valluvaparidasan V, Ladhalakshmi D, Velazhahan R. 2010. Management of bacterial blight of cotton using a mixture of Pseudomonas fluorescens and Bacillus subtilis. Plant Protection Science. 46:41-50.

30. Schaad NW, Jones JB, Chun W (eds). 2001. "Laboratory Guide for Identification of Plant Pathogenic Bacteria. 3nd edition." (APS Press. St. Minnesota, USA. 373pp).

31. Shivalingaiah S, Umesh S. 2013. Pseudomonas fluorescens inhibits the Xanthomonas oryzae pv. oryzae, the bacterial leaf blight pathogen in rice. Canadian Journal of Plant Protection. 1:147-153.

32. Sotami M, Laduc MT, Venkateswaran, K. 2006. Bacillus safensis sp. nov. isolated from spacecraft and assembly facility surfaces. International Journal of Systematic and Evolutionary Microbiology. 56:1735-1740.

33. Suresh A, Pallavi P, Srinivas P, Kumar VP, Chandra SJ. 2010. Plant growth promoting activities of fluorescent pseudomonads associated with some crop plants. African Journal of Microbiology. Research. 4:491-1494.

34. VanPeer R, Schippers B. 1988. Plant growth response in bacterization with selected Pseudomonas spp. strains and rhizosphere microbial development in hydroponic cultures. Canadian Journal of Microbiology. 35:456-463.

\section{Figures}




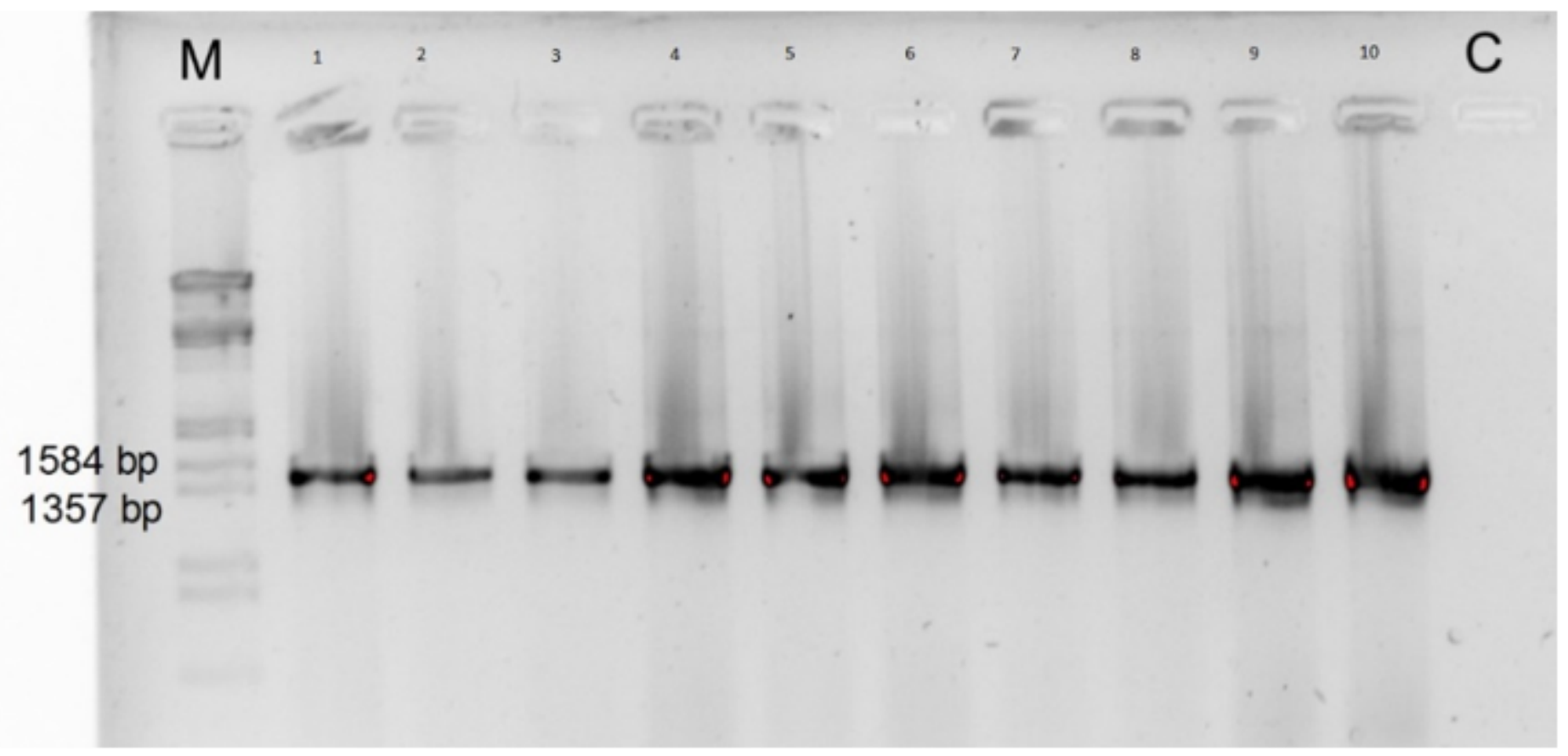

\section{Figure 1}

Amplification of 1500 bp fragment of 16S rRNA gene in PCR. M: Molecular mass indicator, C: Negative control, 1: MR31, 2 \& 3: MR21, 4:MR22, 5 \&6: MR11, 7 \& 8: MR12 and 10: MR13

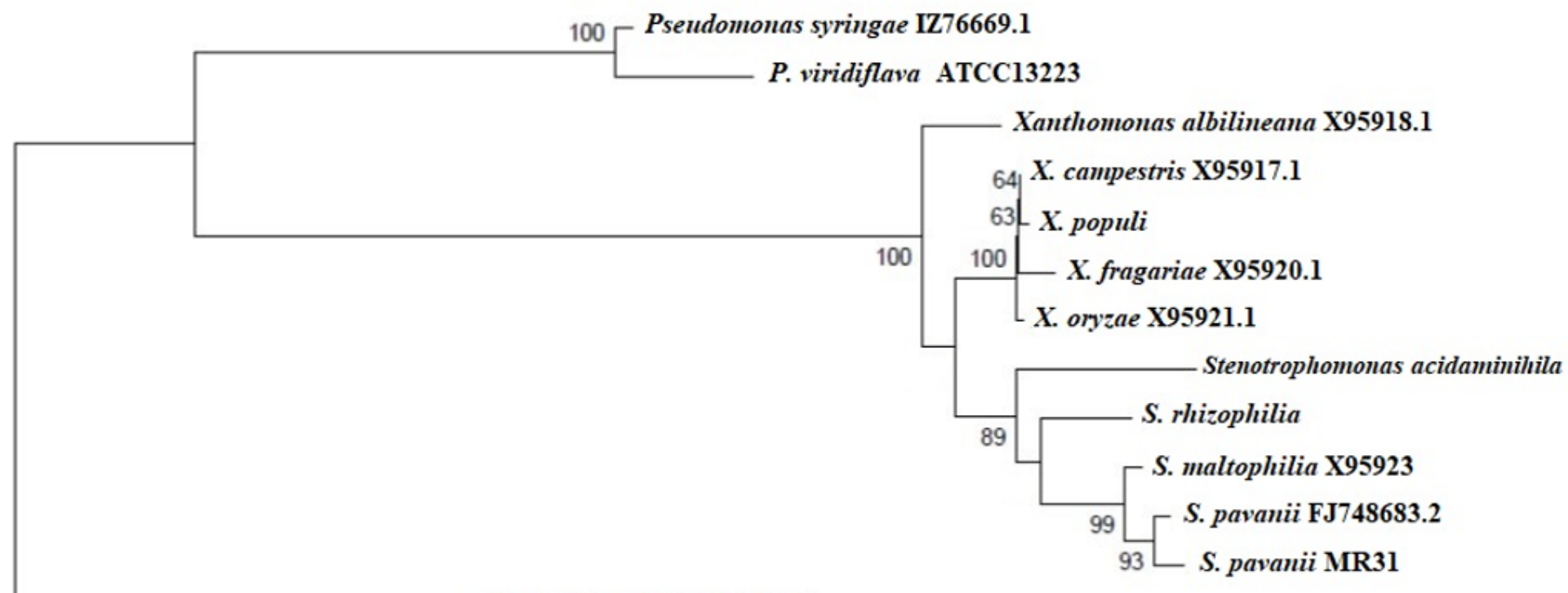

Eschericia coli AF4033733.1

\section{Figure 2}

Phylogenetic relationship of S. pavanii MR31 with other identified strains based on the sequence of 16S rRNA-marker gene. Bootstrap values are shown at the nodes. The tree was rooted with Eschericia coli. 


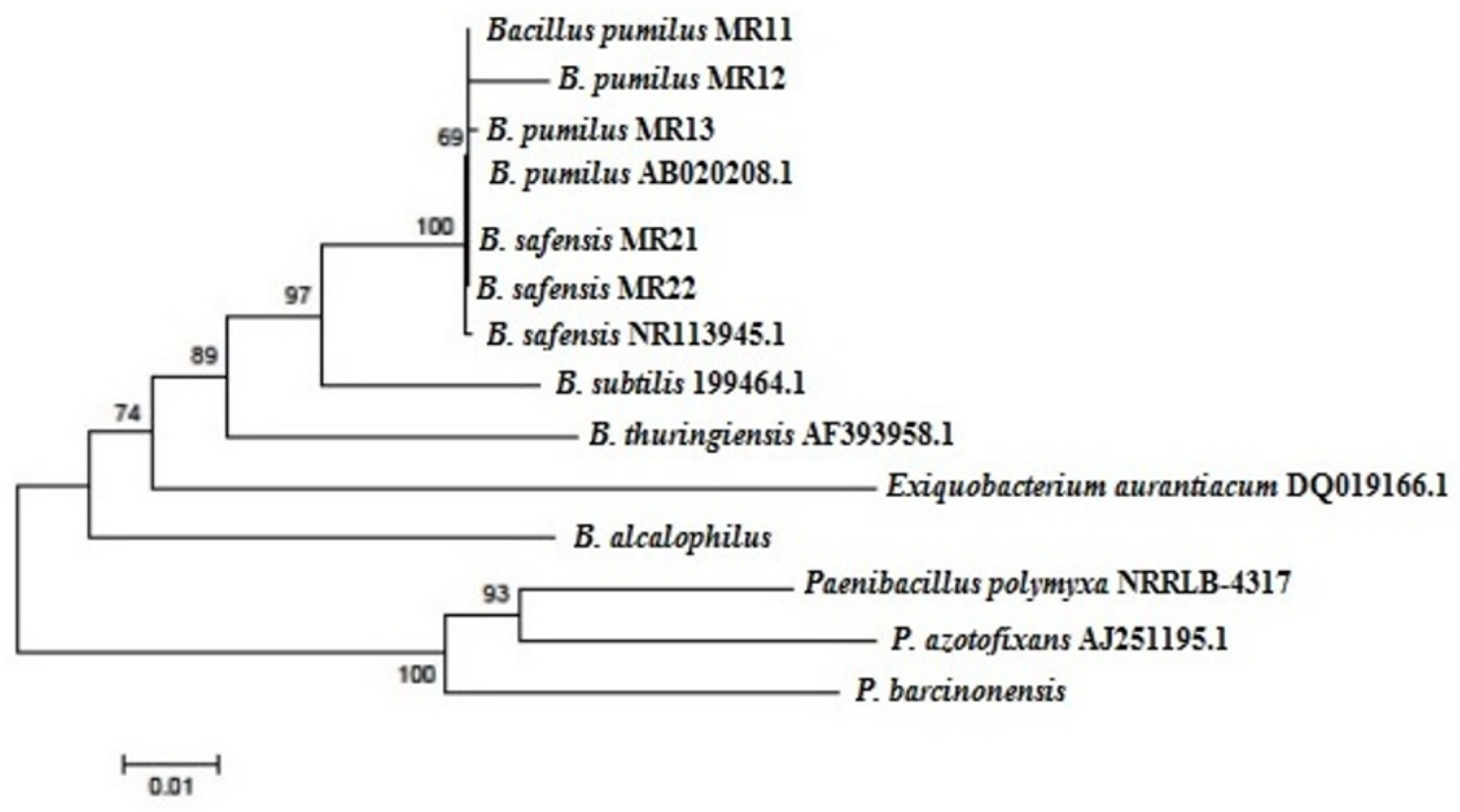

Figure 3

Phylogenetic relationship of B. pumilus MR11, B. pumilus MR12, B. pumilus MR13, B. safensis MR21 and B. safensis MR22 with other identified strains based on the sequence of 16S rRNA-marker gene. Bootstrap values are shown at the nodes. The tree was rooted with Paenibacillus barcinonensis.

\section{Supplementary Files}

This is a list of supplementary files associated with this preprint. Click to download.

- GraphicalAbstract.png

- renamed6bb62.xls 